\title{
Clinicopathological impacts of DNA methylation alterations on pancreatic ductal adenocarcinoma: prediction of early recurrence based on genome-wide DNA methylation profiling
}

\author{
Yutaka Endo ${ }^{1,2} \cdot$ Mao Fujimoto $^{1} \cdot$ Nanako Ito $^{1} \cdot$ Yoriko Takahashi $^{3} \cdot$ Minoru Kitago $^{2} \cdot$ Masahiro Gotoh $^{4}$. \\ Nobuyoshi Hiraoka ${ }^{5} \cdot$ Teruhiko Yoshida $^{4} \cdot$ Yuko Kitagawa $^{2} \cdot$ Yae Kanai $^{1} \cdot$ Eri Arai $^{1} \mathbb{C}$
}

Received: 29 September 2020 / Accepted: 19 January 2021 / Published online: 26 February 2021

(c) The Author(s) 2021

\begin{abstract}
Purpose The present study was conducted to clarify the clinicopathological impacts of DNA methylation alterations on pancreatic ductal adenocarcinoma (PDAC).

Methods Genome-wide DNA methylation screening was performed using the Infinium HumanMethylation450 BeadChip, and DNA methylation quantification was verified using pyrosequencing. We analyzed fresh-frozen tissues from an initial cohort (17 samples of normal control pancreatic tissue [C] from 17 patients without PDAC, and 34 samples of non-cancerous pancreatic tissue [N] and 82 samples of cancerous tissue [T] both obtained from 82 PDAC patients) and formalin-fixed paraffin-embedded $\mathrm{T}$ samples from 34 patients in a validation cohort.

Results The DNA methylation profiles of $\mathrm{N}$ samples tended to differ from those of $\mathrm{C}$ samples, and 91,907 probes showed significant differences in DNA methylation levels between $\mathrm{C}$ and $\mathrm{T}$ samples. Epigenetic clustering of $\mathrm{T}$ samples was significantly correlated with a larger tumor diameter and early recurrence (ER), defined as relapse within 6 months after surgery. Three marker CpG sites, applicable to formalin-fixed paraffin-embedded surgically resected materials regardless of their tumor cell content, were identified for prediction of ER. The sensitivity and specificity for detection of patients belonging to the ER group using a panel combining these three marker $\mathrm{CpG}$ sites, including a CpG site in the CDK14 gene, were 81.8\% and $71.7 \%$ and $88.9 \%$ and $70.4 \%$ in the initial and validation cohorts, respectively.

Conclusion These findings indicate that DNA methylation alterations may have a clinicopathological impact on PDAC. Application of our criteria will ultimately allow prediction of ER after surgery to improve the outcome of PDAC patients.
\end{abstract}

Keywords DNA methylation $\cdot$ Infinium assay $\cdot$ Ductal adenocarcinoma $\cdot$ Pancreas $\cdot$ Early recurrence

Eri Arai

earai@keio.jp

1 Department of Pathology, Keio University School of Medicine, 35 Shinanomachi, Shinjuku-ku, Tokyo 160-8582, Japan

2 Department of Surgery, Keio University School of Medicine, Tokyo 160-8582, Japan

3 Bioscience Department, Solution Knowledge Center, Mitsui Knowledge Industry Co., Ltd., Tokyo 105-6215, Japan

4 Fundamental Innovative Oncology Core Center, National Cancer Center Research Institute, Tokyo 104-0045, Japan

5 Department of Pathology and Clinical Laboratory, National Cancer Center Hospital, Tokyo 104-0045, Japan

$\begin{array}{ll}\text { Abbreviations } \\ \text { AUC } & \text { Area under the curve } \\ \text { BAC } & \text { Bacterial artificial chromosome } \\ \text { C } & \text { Normal control pancreatic tissue } \\ \text { CA19-9 } & \text { Carbohydrate antigen 19-9 } \\ \text { CT } & \text { Computed tomography } \\ \text { DNMT } & \text { DNA methyltransferase } \\ \text { ER } & \text { Early recurrence } \\ \text { GEO } & \text { Gene Expression Omnibus } \\ \text { N } & \text { Non-cancerous pancreatic tissue } \\ \text { ICGC } & \text { International Cancer Genome Consortium } \\ \text { OS } & \text { Overall survival } \\ \text { PCA } & \text { Principal component analysis } \\ \text { PDAC } & \text { Pancreatic ductal adenocarcinoma } \\ \text { ROC } & \text { Receiver-operating characteristic } \\ \text { RFS } & \text { Recurrence-free survival } \\ \text { T } & \text { Cancerous tissue }\end{array}$


TCGA The Cancer Genome Atlas

TSS Transcription start site

\section{Introduction}

Pancreatic ductal adenocarcinoma (PDAC) is a devastating disease that has become one of the major causes of cancerrelated death in the United States (Siegel et al. 2016) and Japan (Egawa et al. 2012). Even in patients with resectable cancer diagnosed early, the cancer recurs after a short interval up to $80 \%$ of cases (Groot et al. 2018). Despite recent developments in pre- and postoperative management (Conroy et al. 2018; Motoi et al. 2019; Murphy et al. 2018; Neoptolemos et al. 2017; Sinn et al. 2017; Uesaka et al. 2016), there is still a significant need to develop more effective strategies, especially those based on molecular profiling, for stratification of patients with PDAC to improve their prognosis.

Early recurrence (ER) of loco-regional and/or distant metastases in PDAC after curative resection has been a serious therapeutic challenge. The median survival time for patients who develop distant metastases within 6 months after surgery is reportedly less than 12 months (Groot et al. 2019; Matsumoto et al. 2015). Therefore, patients in this group with more aggressive PDACs need to be identified before relapse occurs. Previous studies have suggested that tumor size, carbohydrate antigen 19-9 (CA19-9), histologic type, lymph-node metastasis, and microvascular or perineurial invasion are predictive factors for recurrence of PDAC after surgery (Groot et al. 2019; Kurahara et al. 2018; Matsumoto et al. 2015; Nishio et al. 2017; Sugiura et al. 2012). However, such factors alone are not yet sufficiently predictive of ER, and an extensive search for molecular-based biomarkers of ER risk in PDAC is warranted.

Aberrant DNA methylation is one of the most important epigenetic alterations occurring during carcinogenesis, resulting in chromosomal instability and altering the expression levels of tumor-related genes in different organs exposed to various carcinogenetic factors (Baylin et al. 2016; Jones et al. 2016; Kuramoto et al. 2017; Makabe et al. 2019; Ohara et al. 2017; Tsumura et al. 2019). With regard to pancreatic carcinogenesis, we have reported that cumulative DNA methylation of tumor-related genes, such as BRCA1, $A P C, C D K N 2 A$, and TIMP3, is associated with overexpression of DNA methyltransferase (DNMT) 1 (Peng et al. 2005), the major DNMT, even in peripheral pancreatic duct epithelia with an inflammatory background (Peng et al. 2006), which may be at the precancerous stage in the context of chronic pancreatitis. The average number of methylated tumor-related genes increases further during progression from well to poorly differentiated ductal adenocarcinoma (Peng et al. 2005), suggesting that DNA methylation alterations continuously participate in multistage pancreatic carcinogenesis. In addition, based on genome-wide DNA methylation analysis using a bacterial artificial chromosome (BAC) array, we have confirmed the feasibility of DNA methylation diagnostics and prognostication of pancreatic cancers (Gotoh et al. 2011). However, such procedures are not easily applicable in a clinical situation because of the need for simultaneous quantification of DNA methylation status at numerous $\mathrm{CpG}$ sites on multiple BAC clones.

Recently, appropriate single-CpG-resolution genomewide DNA methylation screening methods, e.g., the Infinium assay (Bibikova et al. 2009), have been introduced for analysis of various human tissue specimens. Although many previous studies using such screening methods, including those based on data from the International Cancer Genome Consortium (ICGC) (https://icgc.org) and The Cancer Genome Atlas (TCGA) (https://www.cancer.gov/about-nci/organizati $\mathrm{on} / \mathrm{ccg} /$ research/structural-genomics/tcga), have revealed the DNA methylation profiles of PDACs (Kim et al. 2015; Liu et al. 2019; Nones et al. 2014; Zhang et al. 2019) and diagnostic criteria based on DNA methylation, especially those applicable to prognostication, are still far from established.

In the present study, to further clarify the clinicopathological impacts of DNA methylation profiles on PDAC, we performed single-CpG-resolution genome-wide DNA methylation screening using the Infinium assay in an initial cohort of 133 fresh-frozen pancreatic tissue samples and a meticulous follow-up survey of outcome. The reliability of our prognostication criteria was then investigated in a validation cohort comprising 36 additional samples of microdissected formalin-fixed paraffin-embedded tissue samples.

\section{Materials and methods}

\section{Patients and tissue samples}

The initial cohort consisted of 34 samples of non-cancerous pancreatic tissue $(\mathrm{N})$ and 82 samples of the corresponding cancerous tissue $(\mathrm{T})$ obtained from specimens that had been surgically resected during pancreatectomy from 82 patients with PDAC at the National Cancer Center Hospital, Tokyo, Japan. For comparison, 17 samples of normal control pancreatic tissue (C) obtained from 17 patients without PDAC who underwent pancreatectomy for metastasis of renal cell carcinoma (1 patient), adenocarcinoma of the papilla of Vater (6 patients), gallbladder ( 3 patients) or bile duct (1 patient), abscess (1 patient), serous cystadenoma (1 patient), mucinous cystadenoma (1 patient), solid-pseudopapillary neoplasm (1 patient) and endocrine tumor ( 1 patient) of the pancreas, and lymphoplasmacytic pancreatitis (1 patient) were examined. The validation cohort consisted of $36 \mathrm{~T}$ samples obtained from specimens that had been surgically 
resected by pancreatectomy from 36 patients with PDAC at Keio University Hospital. This study was approved by the Ethics Committees of the National Cancer Center and Keio University, and was performed in accordance with the Declaration of Helsinki. All the patients provided written informed consent before enrollment in the study.

None of the patients in any of the cohorts received preoperative treatment. Preoperative radiological findings were based on the last computed tomography (CT) scan before surgery. Histological diagnosis of surgically resected specimens was made in accordance with the World Health Organization classification (Hruban et al. 2019). All the tumors were classified according to the Tumor-Node-Metastasis Classification of the International Union Against Cancer (Brierley et al. 2017). The clinicopathological parameters of patients belonging to each cohort are summarized in Table S1.

After surgery, PDAC patients usually attended for followup visits with laboratory evaluations every 3 months and CT scans every 6 months for the first 2 years. The patients in the initial cohort did not receive any adjuvant therapy, whereas an oral fluoropyrimidine derivative, $\mathrm{S}-1$, was prescribed to some of the validation cohort patients after surgery. Recurrence was diagnosed by clinicians on the basis of physical examination and imaging modalities such as CT, magnetic resonance imaging, or positron emission tomography, and sometimes confirmed pathologically by biopsy. The followup period for the initial and validation cohorts ranged from 92 to 4578 days (mean, 642.5 days) and from 87 to 909 days (mean, 418.5 days), respectively, after surgery.

\section{Tissue preparation and bisulfite modification}

Tissue specimens in the initial cohort $(17 \mathrm{C}, 34 \mathrm{~N}$ and $82 \mathrm{~T}$ samples) were frozen immediately after surgery and preserved in tanks of liquid nitrogen at the National Cancer Center Biobank, Tokyo, Japan, and those in the validation cohort (36 T samples) were fixed with $10 \%$ neutral-buffered formalin and embedded in paraffin, all in accordance with the Japanese Society of Pathology Guidelines for the Handling of Pathological Tissue Samples for Genomic Research (Kanai et al. 2018). From fresh-frozen tissue samples in the initial cohort, high-molecular-weight DNA was extracted using phenol-chloroform followed by dialysis. We performed microdissection of each sample of formalin-fixed paraffin-embedded tissue in the validation cohort: areas showing a tumor cell content of more than $80 \%$ were dissected using a toothpick under a microscope, avoiding contamination with stromal cells such as infiltrating inflammatory cells and fibroblasts, and non-cancerous epithelial cells. Subsequently, the genomic DNA was extracted from the microdissected specimens using a GeneRead FFPE Kit (QIAGEN GmbH, Hilden, Germany), and 500 and $100 \mathrm{ng}$ of genomic DNA from fresh-frozen tissue samples and microdissected formalin-fixed paraffin-embedded tissue samples, respectively, were subjected to bisulfite treatment using an EpiTect Bisulfite Kit (QIAGEN), in accordance with the manufacturer's protocol.

\section{Infinium assay}

After bisulfite treatment, the DNA methylation status at 485,764 probe sites was examined at single-CpG resolution using the Infinium HumanMethylation450K BeadChip (Illumina, San Diego, CA), in accordance with the manufacturer's instructions. Hybridization fluorescence signals were read with an iScan reader (Illumina). The data were assembled using GenomeStudio methylation software (Illumina). At each $\mathrm{CpG}$ site, the ratio of the fluorescent signal was measured using a methylated probe relative to the sum of the methylated and unmethylated probes, i.e., the so-called $\beta$-value, which ranges from 0.00 to 1.00 , representing the fully unmethylated and fully methylated values for an individual $\mathrm{CpG}$ site, respectively. The results of Infinium assay have been deposited in the Gene Expression Omnibus (GEO) database (https://www.ncbi.nlm.nih.gov/geo/) (accession number: GSE155353).

\section{DNA methylation quantification by pyrosequencing}

The PCR and sequencing primers were designed using PSQ Assay Design Software Version 1.0.6 (Biotage, Uppsala, Sweden). To overcome any PCR bias, we optimized the PCR conditions: $0 \%, 50 \%$, and $100 \%$ of the fully methylated control DNA (Epitect methylated human control DNA, QIAGEN) were used as a template to test the linearity of the protocol, as described previously (Fujimoto et al. 2020; Nagashio et al. 2011). The optimized PCR conditions, i.e., PCR cycle and DNA polymerase, for each primer set are summarized in Table S2. The biotinylated PCR product was captured on streptavidin-coated beads. Quantitative sequencing was performed on a PyroMark Q24 (QIAGEN) using the Pyro Gold Reagents (QIAGEN) in accordance with the manufacturer's protocol. The experiment was conducted in duplicate and the mean DNA methylation level was used as a quantitative value for each sample. When the difference between two measurements was more than $10 \%$, the mean DNA methylation level determined from triplicate experiments was used as a quantitative value.

\section{Immunohistochemistry}

Five-micrometer-thick sections of formalin-fixed paraffinembedded tissue specimens in the validation cohort were deparaffinized and dehydrated. All sections were incubated with anti-human CDK14 rabbit polyclonal antibody 
(HPA015267, Atlas Antibodies AB, Bromma, Sweden; dilution 1:50). Before incubation, the sections were heated for $40 \mathrm{~min}$ at $70{ }^{\circ} \mathrm{C}$ in a water bath using citrate buffer at $\mathrm{pH}$ 6 (Genostaff Co., Ltd., Tokyo, Japan) for antigen retrieval. Endogenous peroxidase activity was blocked by $0.3 \%$ hydrogen peroxide in methanol. Non-specific reactions were blocked with 2.5\% normal horse serum (Vector Laboratories, Inc., Burlingame, CA). The primary antibody incubation was conducted at $4{ }^{\circ} \mathrm{C}$ overnight, followed by incubation with VECTOR ImmPRESS HRP reagent anti-rabbit IgG (MP-7401, Vector Laboratories, Inc.) at room temperature for $30 \mathrm{~min}$. 3.3'-Diaminobenzidine tetrahydrochloride was used as the chromogen. All sections were counterstained with hematoxylin.

Strong CDK14 immunoreactivity was detected in the nucleus in a proportion of cancer cells, whereas slight immunoreactivity was observed in the cytoplasm of most cancer cells. Therefore, distinct nuclear immunoreactivity was considered as positive and the incidence of nuclear CDK14 immunoreactivity was quantitatively evaluated. For each sample in the validation cohort $(n=36), 5$ areas including at least 200 (not more than 300) cancer cells were randomly counted. The incidence of positive immunoreactivity in each area was expressed as a percentage of all the cells counted. The incidence in each sample $(n=36)$ was calculated by taking the average value for all of the 5 areas.

\section{Statistics}

In the Infinium assay, the call proportions $(P$ values for detection of signals above the background $<0.01$ ) for 809 probes in all of the 133 tissue samples of the initial cohort were less than $90 \%$. Since such a low proportion may have been attributable to polymorphism at the probe $\mathrm{CpG}$ sites, these 809 probes were excluded from the present assay, as described previously (Kuramoto et al. 2017; Ohara et al. 2017). In addition, all 11,648 probes on chromosomes $X$ and $\mathrm{Y}$ were excluded to avoid any gender-specific methylation bias, leaving a final total of 473,457 autosomal $\mathrm{CpG}$ sites.

The DNA methylation profiles of the initial cohort $(17 \mathrm{C}$, $34 \mathrm{~N}$, and $82 \mathrm{~T}$ samples) were analyzed using principal component analysis (PCA). Hierarchical clustering (Ward's linkage using Euclidean distances) was performed based on Infinium data for $82 \mathrm{~T}$ samples in the initial cohort. Infinium probes showing significant differences in DNA methylation levels between sample groups were defined by Welch's $t$ test. The associations between clinicopathological parameters and DNA methylation alterations were evaluated using Fisher's exact test. A receiver-operating characteristic (ROC) curve was generated for discriminating the ER group from the non-ER group, and the Youden index for each probe was used as a cut-off value for prediction of ER. All statistical analyses were performed using the statistical program
RStudio (RStudio Inc., Boston, MA) (https://www.rstud io.com), the R software package (R Foundation for Statistical Computing) (https://www.r-project.org), and JMP 12 (SAS Institute Inc., Cary, NC).

\section{Results}

\section{DNA methylation profiles based on Infinium assay}

The Infinium assay identified 91,907 probes that were aberrantly methylated in the $82 \mathrm{~T}$ samples of the initial cohort $(P$ value $<0.05$ by Welch's $t$ test with Bonferroni correction and $\Delta \beta_{\text {T-C }}$ value $>0.1$ or $<-0.1$ ) compared to the $17 \mathrm{C}$ samples, indicating that DNA methylation alterations had occurred in $\mathrm{T}$ samples and participate in pancreatic carcinogenesis. Among the 91,907 probes, 59,900 showed DNA hypermethylation in T samples relative to $\mathrm{C}$ samples, whereas 32,007 probes showed DNA hypomethylation in $\mathrm{T}$ samples relative to $\mathrm{C}$ samples. PCA of $17 \mathrm{C}$ samples, $34 \mathrm{~N}$ samples, and $82 \mathrm{~T}$ samples of the initial cohort using the 91,907 probes indicated that the DNA methylation profile of $\mathrm{N}$ samples tended to differ from that of $\mathrm{C}$ samples (Fig. 1a). Furthermore, the DNA methylation profile of $\mathrm{T}$ samples clearly differed from that of both $\mathrm{C}$ and $\mathrm{N}$ samples (Fig. 1a).

Subsequently, we focused on the N-to-T transition stage during carcinogenesis and identified 66,789 probes that were aberrantly methylated in the $82 \mathrm{~T}$ samples of the initial cohort ( $P$ value $<0.05$ by Welch's $t$ test with Bonferroni correction and $\Delta \beta_{\mathrm{T}-\mathrm{N}}$ value $>0.1$ or $\left.<-0.1\right)$ compared to the $34 \mathrm{~N}$ samples. Among the 66,789 probes, 43,184 showed DNA hypermethylation in $\mathrm{T}$ samples relative to $\mathrm{N}$ samples, whereas 23,605 probes showed DNA hypomethylation in $\mathrm{T}$ samples relative to $\mathrm{N}$ samples. Using the 66,789 probes, hierarchical clustering (Ward's linkage using Euclidean distances) of $82 \mathrm{~T}$ samples was performed (Fig. 1b): $82 \mathrm{~T}$ samples were clustered into four subclasses: Clusters A $(n=10)$, B1 $(n=8), \mathrm{B} 2(n=16)$, and B3 $(n=48)$. The clinicopathological parameters of the epigenetic clusters are summarized in Table S3A. Although Table S3A shows a significant association between epigenetic clustering and lymphovascular invasion, a low incidence of lymphovascular invasion was restricted to the smallest Cluster B1 $(n=8)$ and such an association appeared to lack any clinical impact. On the other hand, Table S3A suggested that Clusters A and B3 shared a tendency for a larger tumor size. Therefore, we decided to combine clusters with similar tendencies into Clusters $\mathrm{A}$ and B3 and Clusters B1 and B2 to highlight the differences in their clinicopathological parameters (Table S3B). PDAC tumors belonging to Clusters A and B3 were significantly larger than those belonging to Clusters B1 and B2 $(P=0.030)$. Moreover, when ER was defined as relapse within 6 months after surgery, all 22 patients showing ER 
(A)

(B)
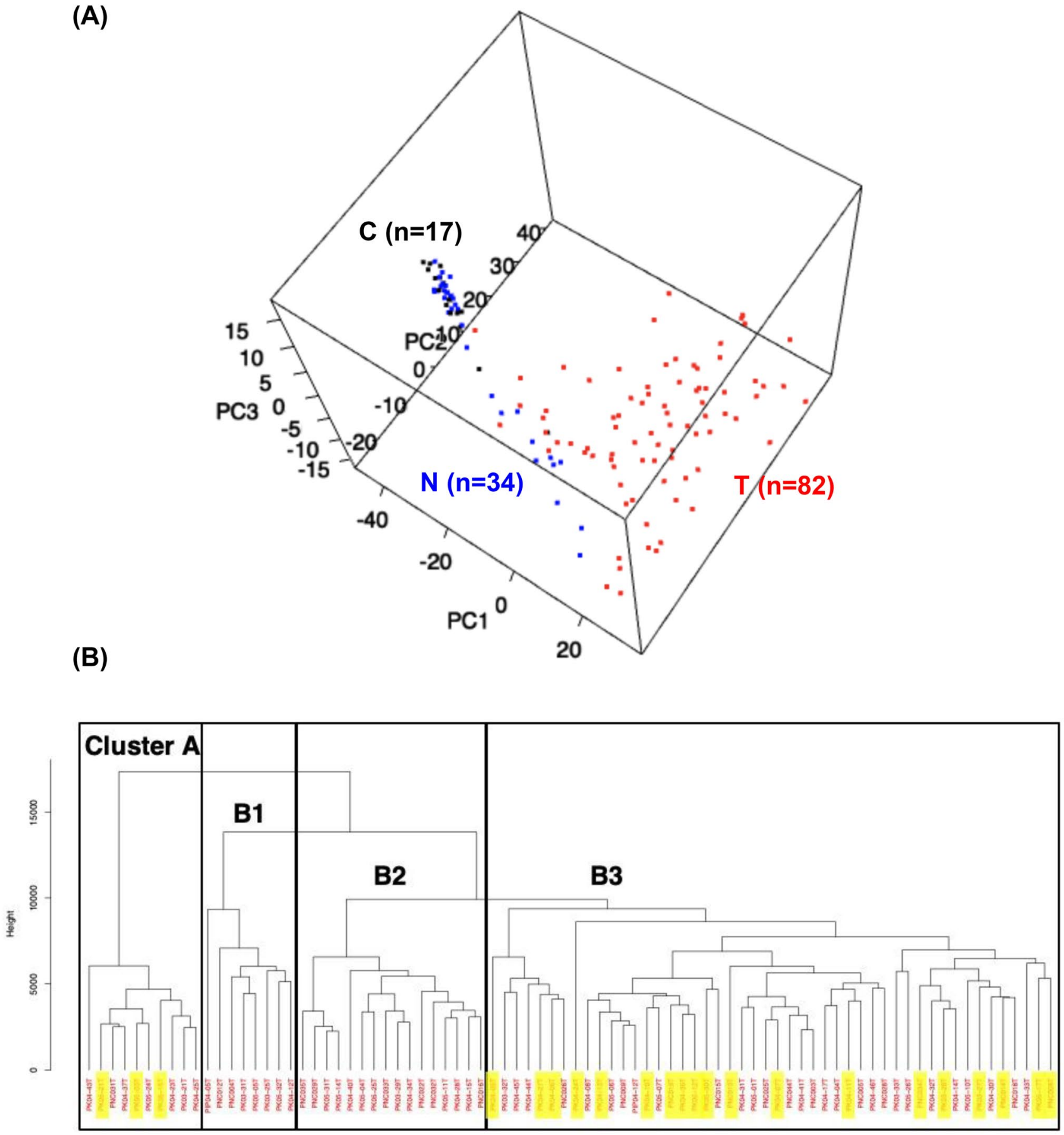

Fig. 1 DNA methylation profiles based on Infinium assay in the initial cohort. a Principal component analysis of all 133 samples (17 C, $34 \mathrm{~N}$ and $82 \mathrm{~T}$ samples) using the 91,907 probes that were aberrantly methylated in the T samples ( $P$ value $<0.05$ by Welch's $t$ test with Bonferroni correction and $\Delta \beta_{\mathrm{T}-\mathrm{C}}$ value $>0.1$ or $\left.<-0.1\right)$ compared to the $\mathrm{C}$ samples. PC, principal component. b Hierarchical clustering (Ward's linkage using Euclidean distances) in $82 \mathrm{~T}$ samples using the 66,789 probes that were aberrantly methylated in $\mathrm{T}$ samples $(P$ value $<0.05$ by Welch's $t$ test with Bonferroni correction and $\Delta \beta_{\mathrm{T}-\mathrm{N}}$ value $>0.1$ or $<-0.1)$ compared to the $\mathrm{N}$ samples. $\mathrm{T}$ samples were clustered into four subclasses: Clusters A $(n=10), \mathrm{B} 1 \quad(n=8), \mathrm{B} 2$ $(n=16)$, and B3 $(n=48)$. Patients showing early recurrence defined as relapse within 6 months after surgery are marked with yellow 
were included in Cluster A or B3, as shown in Fig. 1b: the incidence of ER in Cluster A or B3 (37.9\%) was significantly higher than that in Clusters B1 and B2 $(0 \%, P=0.0002)$.

\section{Identification of marker CpG sites for discrimination of the ER group}

At the time of last follow-up in the initial cohort $(n=82)$, 71 patients $(86.6 \%)$ had suffered disease recurrence after a median recurrence-free survival (RFS) period of 12.4 months. There were no significant differences in any of the clinicopathological parameters reflecting tumor aggressiveness between the ER group $(n=22)$ and the nonER group who suffered recurrence 6 months or later after surgery $(n=60)$ (Table S4), indicating that the risk of ER cannot be predicted based on such parameters, including the level of the widely used serum tumor marker CA199. Median overall survival (OS) for the entire cohort was 35.6 months. On the other hand, OS for the ER group was 11.2 months, whereas that for the non-ER group was 48.9 months (Fig. $2, P<0.0001$ by Log-rank test), indicating the importance of ER prediction for deciding the follow-up strategy for patients with PDAC to improve their outcome.

Since our Infinium assay indicated that DNA methylation profiles have some impact on the aggressiveness of PDAC (Fig. 1b and Table S3B), we attempted to establish diagnostic criteria for prediction of ER based on DNA methylation. Welch's $t$ test identified 134 probes showing significant differences in DNA methylation levels between the ER and non-ER groups ( $P$ value $<0.05$ and $\Delta \beta_{\text {ER-non-ER }}$ value $>0.1$ or $<-0.1$ ). Next, using these 134 probe $\mathrm{CpG}$ sites, the ROC curves were generated to discriminate patients belonging to the ER group from patients belonging to the non-ER group. Among the $134 \mathrm{CpG}$ sites, 58 showed AUC values of more than 0.7 and are summarized in Table S5. Among them, scattergrams of DNA methylation levels for the 14 representative $\mathrm{CpG}$ sites marked by asterisks in Table $\mathrm{S} 5$ are shown in Fig. 3, indicating that these $14 \mathrm{CpG}$ sites could be candidate markers for discrimination of the ER group.

\section{Pyrosequencing for technical verification of DNA methylation levels of candidate marker CpG sites}

The Infinium assay is generally considered to be a genomewide screening method, whereas pyrosequencing is a precise method for quantification of DNA methylation at specific CpG sites. In fact, when we preliminarily established ER prediction criteria based solely on Infinium data for the marker CpG sites included in Table S5 and Fig. 3, the overall diagnostic impact was insufficient (data not shown). In addition, pyrosequencing of individual marker $\mathrm{CpG}$ sites is more suitable in a clinical laboratory situation. Therefore, we decided to establish our ER prediction criteria based on pyrosequencing.

Although we intended to technically verify the DNA methylation levels of the 14 candidate marker $\mathrm{CpG}$ sites
Fig. 2 Overall survival of patients in the initial cohort. When early recurrence (ER) was defined as relapse within 6 months after surgery, patients belonging to the ER group $(n=22)$ showed a poorer outcome than patients belonging to the non-ER group $(n=60)$ $(P<0.0001$ by Log-rank test $)$

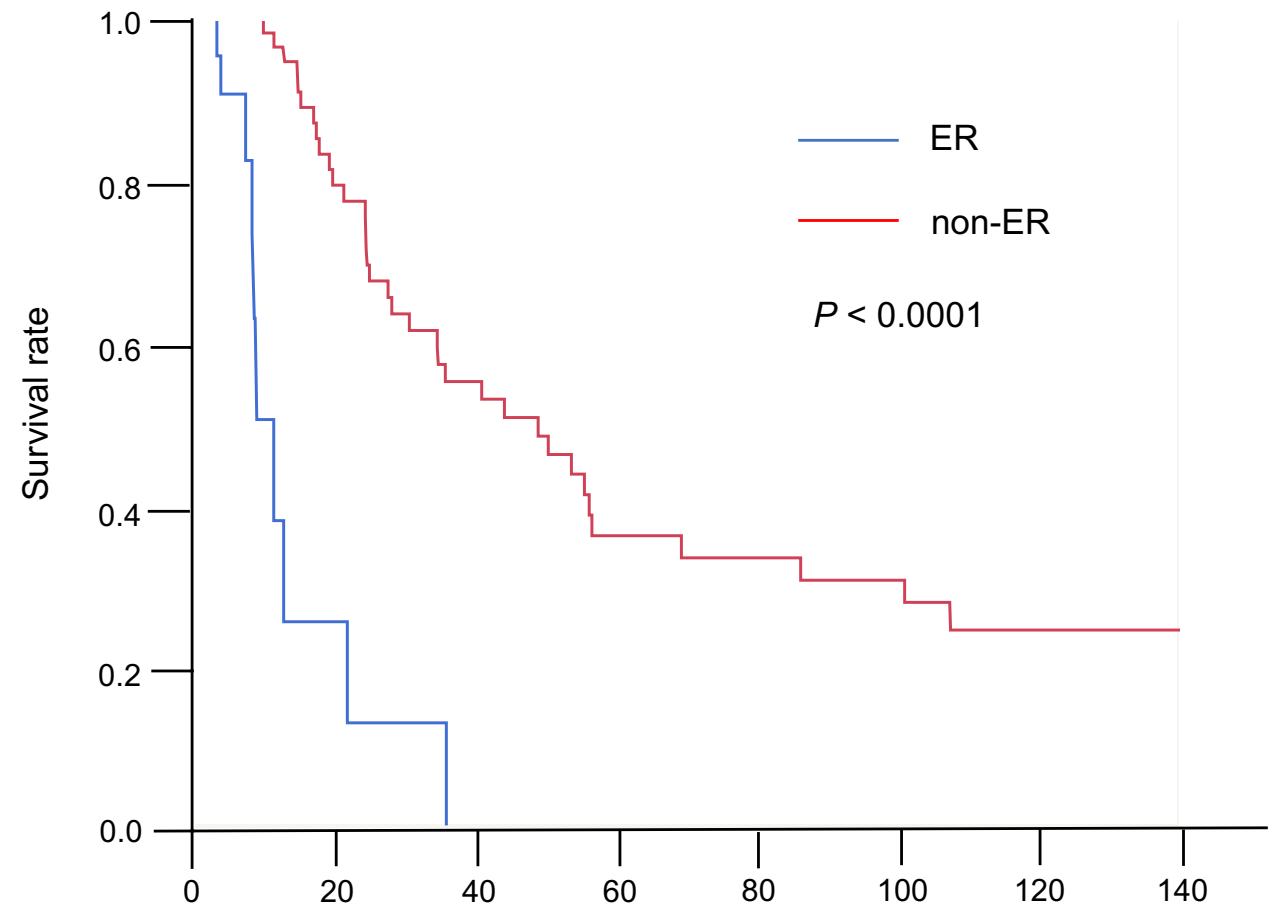

Time after operation (months) 
using pyrosequencing, optimization of the PCR conditions was very difficult for pyrosequencing of 2 of the 14 CpG sites (cg21867733, cg02627240). On the other hand, the PCR conditions for pyrosequencing were successfully optimized for verification of the remaining 12 candidate marker CpG sites (cg00945409, cg07846168, cg17206555, cg02046247, cg18289710, cg09229620, cg15930703, cg21873275, cg02192855, cg19918599, cg20549290, and cg14064694) (Table S2): the linearity of the measured values and their consistency with the theoretical values for the 12 CpG sites are shown in Figure S1.

The DNA methylation levels of the $12 \mathrm{CpG}$ sites were quantified using pyrosequencing in the initial cohort, and all were found to be significantly correlated with those obtained using the Infinium assay (Pearson correlation coefficient $[\mathrm{r}] \geq 0.801$ and $P<0.0001$ ) (Figure S2), indicating that the Infinium data had been successfully verified by pyrosequencing and that the $12 \mathrm{CpG}$ sites identified on the basis of the Infinium assay were valid prognostic markers. Table 1 summarizes the gene names, chromosomes, $\mathrm{CpG}$ types (islands, island shores [2000-bp regions adjacent to a CpG island], and island shelves [2000-bp regions adjacent to an island shore] based on the University of California, Santa Cruz [UCSC] genome browser [https://genome.ucsc. edu/]) and annotations (TSS1500 [from $200 \mathrm{bp}$ upstream of the transcription start site [TSS] to $1500 \mathrm{bp}$ upstream of it], TSS200 [from TSS to $200 \mathrm{bp}$ upstream of it], 5' UTR [untranslated region], 1st exon, 1st intron, and gene body [2nd exon and downstream] identified using the RefSeq database [http://www.ncbi.nlm.nih.gov/refseq/]) for the 12 CpG sites.

Table 1 included a $\mathrm{CpG}$ island, island shores, and a shelf around TSS, i.e., TSS200, TSS1500, 1st exon, and 1st intron, which are considered to be critical for regulating the expression of specific genes. On the other hand, not a few surrogate marker $\mathrm{CpG}$ sites were identified even in open sea regions in the gene bodies (Table 1), suggesting that $\mathrm{CpG}$ sites regulating the expression of tumor-related genes potentially participating in tumor aggressiveness, including ER, and those that may not participate in such gene expression regulation but are probably included in the domains that are controlled in parallel with the level of DNA methylation of such tumorrelated genes, could be used as ER predictors.

\section{Establishment of criteria for prediction of ER}

To establish criteria for prediction of ER using each of the 12 marker $\mathrm{CpG}$ sites, ROC curves were generated using the pyrosequencing data for discriminating patients belonging to the ER group from those belonging to the non-ER group (Figure S3), and Youden indices were set as cut-off values for each $\mathrm{CpG}$ site. Even when pyrosequencing data were used, AUC values of $>0.7$ were again obtained for each of the 12 exact Infinium probe $\mathrm{CpG}$ sites (Table 2).

When neighboring $\mathrm{CpG}$ sites other than exact Infinium probe $\mathrm{CpG}$ sites are located within amplicons for pyrosequencing, the DNA methylation levels of such neighboring sites are simultaneously quantified by pyrosequencing. For example, in addition to the exact Infinium probe cg00945409 site (position 1), DNA methylation data for a neighboring CpG site (position 2) were obtained, as shown in Table S2. Among these 13 neighboring $\mathrm{CpG}$ loci, 10 also showed AUC values of $>0.7$ for discriminating the ER group (Figure S3) and are included in Table 2. Using each of the 22 diagnostic criteria (12 exact Infinium probe $\mathrm{CpG}$ sites and their 10 neighboring $\mathrm{CpG}$ sites), the sensitivity for diagnosis of patients showing ER $(n=22)$ in the initial cohort patients overall $(n=82)$ ranged from 40.9 to $100 \%$, and the specificity from 40.0 to $95.0 \%$ (Table 2).

\section{Confirmation of criteria reliability using the validation cohort}

At the time of the last follow-up of the validation cohort $(n=36), 20(55.6 \%)$ patients had suffered recurrence after a median RFS of 16.3 months. There were nine patients (25.0\%) in the ER group: the incidence of ER in the validation cohort did not differ significantly from that in the initial cohort. To validate the reliability of our criteria for diagnosis of ER, pyrosequencing was performed in the validation cohort using the cut-off values, as shown in Table 2. For three candidate CpG sites (cg07846168, cg09229620, and cg20549290), sufficient PCR products were not obtained. Finally, it was confirmed that both the sensitivity and specificity at three marker CpG sites-cg 17206555 (position 1), $\operatorname{cg} 02046247$ (position 1), and 19,918,599 (position 2)—were more than $59 \%$ in the validation cohort.

Subsequently, a diagnostic panel was established by combining these three marker $\mathrm{CpG}$ sites, as shown in Table 3 . When two or more $\mathrm{CpG}$ sites satisfied the criteria, as shown in Table 2, sufficient sensitivity and specificity for identification of the ER group patients were obtained in both the initial $(81.8 \%$ and $71.7 \%)$ and validation $(88.9 \%$ and $70.4 \%$ ) cohorts, respectively (Table 3 ). If higher sensitivity is needed before intensive screening of recurrent and/or metastatic lesions, positivity for only one of three $\mathrm{CpG}$ sites may be adequate. On the other hand, if higher specificity is needed before adjuvant therapy, positivity for all three $\mathrm{CpG}$ sites may be adequate as criteria.

\section{Immunohistochemistry for CDK14 expression in PDACs}

Since DNA hypomethylation of the CDK14 (cyclindependent kinase 14) gene was an excellent marker for ER 

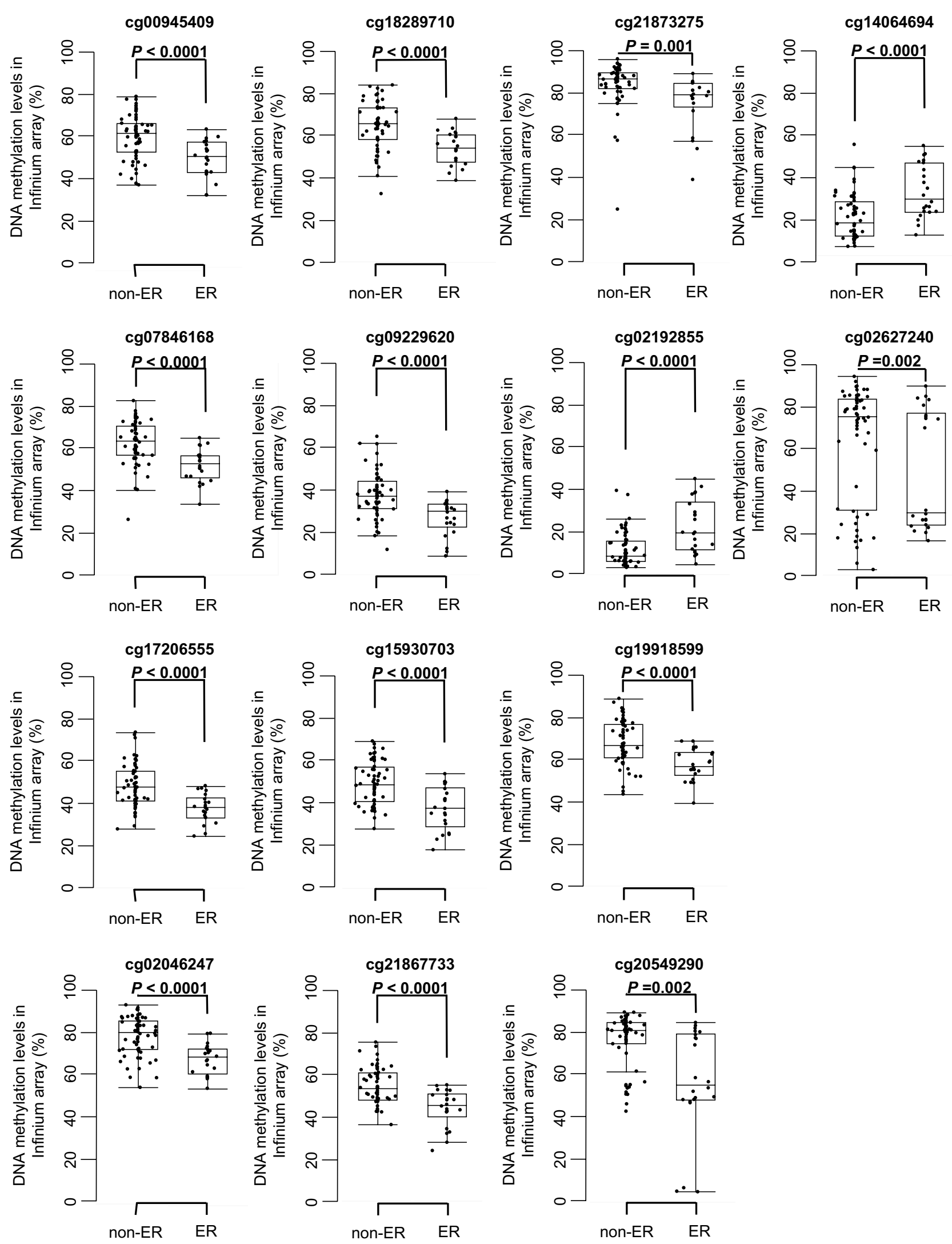
4Fig. 3 DNA methylation levels obtained by Infinium assay of candidate marker $\mathrm{CpG}$ sites in tissue samples in the early recurrence (ER) group $(n=22)$ and the non-ER group $(n=60)$ in the initial cohort. Infinium probe IDs and $P$ values in Welch's $t$ test are shown in each panel. DNA methylation levels for each of the Infinium probes, $\operatorname{cg} 14064694$ and $\operatorname{cg} 02192855$, in the ER group are significantly higher than those in the non-ER group. The DNA methylation levels for each of the Infinium probes, cg00945409, cg18289710, cg21873275, cg07846168, cg09229620, cg02627240, cg17206555, cg15930703, cg19918599, cg02046247, cg21867733, and cg20549290, in the ER group are significantly lower than those in the non-ER group

prediction (Table 3), CDK14 protein expression was examined by immunohistochemistry. Representative photos of CDK14-negative and CDK14-positive areas of PDACs are shown in Figure S4A. As shown in Figure S4B, the incidence of nuclear immunoreactivity for CDK14 in PDACs belonging to the ER group $(n=9)$ is significantly higher than that in PDACs belonging to the non-ER group $(n=27)$ in the validation cohort $(P=0.0020)$, indicating the possibility that the level of nuclear CDK14 expression could be used as an ER predictor.

\section{Discussion}

Based on the present PCA by Infinium assay, the DNA methylation profile of $\mathrm{N}$ samples, which were obtained from patients with PDAC and may already have been exposed to carcinogenetic factors, tended to differ from that of $\mathrm{C}$ samples (Fig. 1a). Not a few $\mathrm{N}$ samples showed chronic pancreatitis, which is widely considered to be one of the precancerous conditions for PDAC (Hassan et al. 2007). Therefore, the differences in DNA methylation profiles between $\mathrm{C}$ and $\mathrm{N}$ samples may have been at least partly attributable to differences in their cellular components: $\mathrm{N}$ samples would have contained more infiltrating lymphocytes and fibroblasts in the background of chronic inflammation. On the other hand, our previous meticulous microdissection technique and immunohistochemistry have revealed that peripheral pancreatic duct epithelial cells, i.e., the origin of ductal adenocarcinoma (Hruban et al. 1997), and not surrounding lymphocytes or fibroblasts, actually show cumulative DNA methylation abnormalities of tumor-related genes associated with aberrant expression of DNMT1 at the precancerous N stages (Peng et al. 2005, 2006). Analogous with the results of these previous studies, the differences in the DNA methylation profiles of $\mathrm{N}$ samples relative to $\mathrm{C}$ samples in the present study may have been at least partly induced in peripheral pancreatic duct epithelial cells themselves by precancerous conditions such as chronic pancreatitis. Such participation of DNA methylation alterations even in the early and precancerous stages of multistage carcinogenesis is consistent with the results of our previous Infinium assay using many samples of cancerous tissue from various organs (Arai et al. 2009, 2012; Sato et al. 2014; Yamanoi et al. 2015).

The DNA methylation profile of T samples clearly differed from that of both $\mathrm{C}$ and $\mathrm{N}$ samples (Fig. 1a). Since, in the initial cohort, Infinium assay was performed using bulk frozen tissue samples, at least some of the differences in DNA methylation profiles between $\mathrm{T}$ and other samples may again have been attributable to differences in their cellular components: $\mathrm{C}$ and $\mathrm{N}$ samples contain not only peripheral pancreatic ducts, the origin of ductal adenocarcinoma (Hruban et al. 1997), but also acinar cells and islet cells. On the other hand, on the PCA scattergram (Fig. 1a), N samples were located nearer to $\mathrm{T}$ samples than to $\mathrm{C}$ samples. This finding is again consistent with the previous results from various organs where DNA methylation alterations at the precancerous stage are inherited by or strengthened in the tumorous tissue themselves (Arai et al. 2009, 2012; Sato et al. 2014; Yamanoi et al. 2015).

When we focused on the DNA methylation profiles of $\mathrm{T}$ samples themselves, it was possible to subclassify pancreatic cancer patients into epigenomic Clusters A and B3 vs Clusters B1 and B2, which were significantly correlated with the clinicopathological aggressiveness of PDAC, i.e., larger tumor size and early recurrence, indicating that DNA methylation abnormalities at least partly determine the malignant potential of cancers, as has been observed in many other organs (Arai et al. 2009, 2012; Sato et al. 2014; Yamanoi et al. 2015). This finding motivated us to perform prognostication of patients with PDAC based on their DNA methylation profiles. Prediction of ER risk after surgery is critical for improving the outcome of PDAC. Although previous studies that separately defined ER as occurring at 6 (Matsumoto et al. 2015), 8 (Nishio et al. 2017), or 12 (Groot et al. 2019) months after surgery, since the present study revealed that patients suffering recurrence within 6 months after curative resection clearly showed poorer survival (Fig. 2), we defined ER as relapse occurring within 6 months after surgery. Although it was not possible to predict ER on the basis of clinicopathological parameters (Table S4), we successfully identified DNA methylation biomarkers for prediction of ER based on technical verification by pyrosequencing and biological validation using the validation cohort. Most previous studies have based prognostication of patients with PDAC on clinicopathological findings (Matsumoto et al. 2015) or perioperative serum markers (Sugiura et al. 2012), and this approach has not yet achieved sufficient predictive accuracy. To our knowledge, this study is the first reported to have employed DNA methylation biomarkers for ER prediction. Unlike alterations of mRNA and protein expression, which can be easily affected by the microenvironment of cancer cells, DNA methylation alterations are stably preserved on DNA double strands by covalent bonds. Therefore, 
Table 1 Potential marker $\mathrm{CpG}$ sites discriminating the early recurrence (ER) group from the non-ER group based on Infinium assay, for which data had been verified using pyrosequencing in the initial cohort

\begin{tabular}{lllll}
\hline Probe ID $^{\mathrm{a}}$ & $\begin{array}{l}\text { Chromo- } \\
\text { some }\end{array}$ & $\begin{array}{l}\text { Gene } \\
\text { symbol }^{\mathrm{b}}\end{array}$ & CpG type $^{\mathrm{c}}$ & Gene region $^{\mathrm{d}}$ \\
\hline $\operatorname{cg} 00945409$ & 10 & ZMIZ1-AS1 & S_Shelf & Gene body \\
$\operatorname{cg} 07846168$ & 13 & GPC6 & Open sea & Gene body \\
$\operatorname{cg} 17206555$ & 7 & CDK14 & S_Shore & First intron \\
$\operatorname{cg} 02046247$ & 12 & NA & Open sea & Intergenic \\
& & & region \\
$\operatorname{cg} 18289710$ & 11 & PDGFD & Open sea & Gene body \\
$\operatorname{cg} 09229620$ & 3 & NLGN1 & S_Shore & $5^{\prime}$ UTR \\
$\operatorname{cg} 15930703$ & 2 & DOCK10 & Open sea & Gene body \\
$\operatorname{cg} 21867733$ & 12 & NCOR2 & Open sea & $5^{\prime}$ UTR \\
$\operatorname{cg} 21873275$ & 1 & NBPF25P & Open sea & Gene body \\
$\operatorname{cg} 02192855$ & 6 & HIST1H2BI & N_Shore & TSS200 \\
$\operatorname{cg} 19918599$ & 3 & NA & Open sea & Intergenic \\
& & & region \\
$\operatorname{cg} 20549290$ & 7 & GIMAP4 & Open sea & TSS1500 \\
$\operatorname{cg} 14064694$ & 6 & HIST1H2BI & Island & First exon \\
$\operatorname{cg} 02627240$ & 7 & TPK1 & Open sea & Gene body \\
\hline
\end{tabular}

a Probe IDs for the Infinium HumanMethylation450 BeadChip (Illumina)

${ }^{\mathrm{b}} \mathrm{NA}$ : not annotated (located within intergenic regions)

${ }^{\mathrm{c}} \mathrm{CpG}$ islands, island shores (2000-bp regions adjacent to a $\mathrm{CpG}$ island), and island shelves (2000-bp regions adjacent to an island shore) are identified based on the University of California, Santa Cruz (UCSC) genome browser (https://genome.ucsc.edu/)

${ }^{\mathrm{d}}$ TSS1500 (from $200 \mathrm{bp}$ upstream of the transcription start site [TSS] to 1500 bp upstream of it), TSS200 (from TSS to $200 \mathrm{bp}$ upstream of it), 5' UTR (untranslated region), first exon, first intron, and gene body (second exon and downstream) are identified based on the RefSeq database (http://www.ncbi.nlm.nih.gov/refseq/)

alterations of DNA methylation would potentially be optimal prognostic indicators for affected patients.

Here, we used formalin-fixed paraffin-embedded surgically resected materials in the validation study after genomewide screening, because usage of such materials is very feasible in a clinical setting. However, the prognostic impact of some candidate marker $\mathrm{CpG}$ sites based on Infinium assay in the initial cohort failed to be verified in the validation cohort. This discrepancy of DNA methylation data between formalin-fixed paraffin-embedded tissue and fresh-frozen tissue was consistent with a previous report by Wen et al. (2017), who suggested that the reasons for such discrepancy might include formaldehyde-induced cross-links, DNA fragmentation, and deamination of cytosine bases. Another reason may be differences in the tumor cell content. Since bulk tissue used in the initial screening had a generally low tumor cell content, the Infinium assay would have potentially highlighted $\mathrm{CpG}$ sites showing DNA methylation abnormalities in fibroblasts and endothelial cells in the stroma of aggressive cancers with a risk of ER. It is known that inflammatory cytokines can affect the DNA methylation status of cells (Mishra 2020). Since infiltrating inflammatory cells secrete inflammatory cytokines, the DNA methylation status of both cancer cells and stromal cells of PDACs derived from a background of chronic pancreatitis might be regulated by the cytokine-rich tumor microenvironment. Various growth factors are known to be produced by both cancer cells and stromal cells, and potentiate cancer cell proliferation and/or invasiveness in an autocrine and paracrine manner. Therefore, it is feasible that the DNA methylation status of both cancer cells and stromal cells is altered, and that such alteration might determine tumor aggressiveness via alterations in the expression levels of such growth factors.

On the other hand, before the validation study, we microscopically dissected areas showing a tumor cell content of more than $80 \%$ from formalin-fixed paraffin-embedded specimens. Therefore, $\mathrm{CpG}$ sites reflecting abnormalities of only non-cancerous stromal cells, such as fibroblasts and endothelial cells, and not those of tumor cells were not verified in the validation study. Finally, after the validation study, we were able to successfully identify $\mathrm{CpG}$ sites that could be applicable as biomarkers regardless of tumor cell content, even using formalin-fixed paraffin-embedded specimens. Table 3 indicates that it would be possible to predict ER by quantifying only three validated marker $\mathrm{CpG}$ sites. Such a small number of quantification targets suggested that this approach would be feasible as a laboratory examination in a clinical setting, as well as being applicable to formalin-fixed paraffin-embedded surgically resected materials.

We additionally tried to identify prognostic markers in Cluster A and Cluster B3 individually. In Cluster A, 484 $\mathrm{CpG}$ sites showing significant differences in DNA methylation levels between the ER and non-ER groups ( $P$ value $<0.05$ and $\Delta \beta_{\text {ER-non-ER value }}>0.1$ or $<-0.1$ ) and AUC values of $>0.7$ were identified. In addition, 143 marker CpG sites were identified in Cluster B3. However, the sensitivity and specificity for all samples in the initial cohort were very low (data not shown), indicating the possibility of overfitting.

Table 3 shows that a $\mathrm{CpG}$ site of DNA hypomethylation of the $C D K 14$ gene was an excellent marker for ER prediction. Since the marker CpG site (position 1 of $\operatorname{cg} 17206555$ ) is located within the CpG island shore around the TSS (Table 1), such DNA hypomethylation would potentially result in overexpression of the gene in aggressive PDAC. Indeed, a significant inverse correlation between DNA methylation and mRNA expression levels $(r=-0.442$, $P<0.0001)$ was confirmed based on data for samples of cancerous and non-cancerous pancreatic tissue $(n=182)$ deposited in the TCGA database (https://www.cancer.gov/about -nci/organization/ccg/research/structural-genomics/tcga) (Figure S5). In fact, our immunohistochemical examination 
Table 2 DNA methylation diagnostics for the early recurrence (ER) group based on pyrosequencing data

\begin{tabular}{|c|c|c|c|c|c|c|c|c|}
\hline \multirow[t]{2}{*}{ Probe $\mathrm{ID}^{\mathrm{a}}$} & \multirow[t]{2}{*}{ Position $^{\mathrm{b}}$} & \multirow[t]{2}{*}{ DNA methylation status ${ }^{c}$} & \multirow[t]{2}{*}{ AUC } & \multirow{2}{*}{$\begin{array}{l}\text { Cut-off value } \\
(\%)\end{array}$} & \multicolumn{2}{|l|}{ Initial cohort } & \multicolumn{2}{|l|}{ Validation cohort } \\
\hline & & & & & Sensitivity $(\%)^{\mathrm{d}}$ & Specificity $(\%)^{\mathrm{e}}$ & Sensitivity $(\%)^{\mathrm{d}}$ & Specificity $(\%)^{\mathrm{e}}$ \\
\hline \multirow[t]{2}{*}{$\operatorname{cg} 00945409$} & 1 & ER $<$ non-ER & 0.778 & 58.6 & 90.9 & 60.0 & 66.7 & 44.4 \\
\hline & 2 & & 0.764 & 71.6 & 86.4 & 68.3 & 22.2 & 88.9 \\
\hline $\operatorname{cg} 07846168$ & 1 & ER $<$ non-ER & 0.791 & 61.3 & 95.5 & 56.7 & NA & NA \\
\hline \multirow[t]{2}{*}{$\operatorname{cg} 17206555$} & 1 & ER $<$ non-ER & 0.811 & 23.5 & 86.4 & 71.7 & 66.7 & 77.8 \\
\hline & 2 & & 0.760 & 18.7 & 86.4 & 65.0 & 55.6 & 74.1 \\
\hline \multirow[t]{2}{*}{$\operatorname{cg} 02046247$} & 1 & ER $<$ non-ER & 0.775 & 79.0 & 90.9 & 60.0 & 88.9 & 63.0 \\
\hline & 2 & & 0.739 & 92.0 & 86.4 & 53.3 & 88.9 & 7.41 \\
\hline $\operatorname{cg} 18289710$ & 1 & ER $<$ non-ER & 0.759 & 61.6 & 72.7 & 70.0 & 77.8 & 22.2 \\
\hline \multirow[t]{2}{*}{$\operatorname{cg} 09229620$} & $\underline{2}$ & ER $<$ non-ER & 0.708 & 41.7 & 77.3 & 56.7 & NA & NA \\
\hline & 3 & & 0.715 & 62.8 & 77.3 & 56.7 & NA & NA \\
\hline \multirow[t]{2}{*}{$\operatorname{cg} 15930703$} & 1 & ER $<$ non-ER & 0.753 & 42.9 & 100 & 40.0 & 88.9 & 14.8 \\
\hline & 2 & & 0.730 & 28.9 & 63.6 & 80.0 & 55.6 & 40.7 \\
\hline cg21873275 & 1 & ER $<$ non-ER & 0.744 & 77.7 & 72.7 & 68.3 & 44.4 & 74.1 \\
\hline \multirow[t]{2}{*}{ cg02192855 } & 1 & ER $>$ non-ER & 0.720 & 13.1 & 50.0 & 93.3 & 22.2 & 63.0 \\
\hline & $\underline{3}$ & & 0.773 & 7.32 & 81.8 & 60.0 & 44.4 & 37.0 \\
\hline \multirow[t]{2}{*}{ cg19918599 } & 1 & ER $<$ non-ER & 0.759 & 77.9 & 86.4 & 58.3 & 77.8 & 55.6 \\
\hline & 2 & & 0.721 & 70.2 & 77.3 & 60.0 & 77.8 & 59.3 \\
\hline \multirow[t]{2}{*}{$\operatorname{cg} 20549290$} & 1 & ER $<$ non-ER & 0.777 & 84.1 & 72.7 & 75.0 & NA & NA \\
\hline & 2 & & 0.739 & 82.0 & 77.3 & 61.7 & NA & NA \\
\hline \multirow[t]{3}{*}{$\operatorname{cg} 14064694$} & 2 & ER $>$ non-ER & 0.714 & 19.0 & 54.6 & 81.7 & 33.3 & 51.9 \\
\hline & $\underline{3}$ & & 0.759 & 9.99 & 95.5 & 45.0 & 77.8 & 29.6 \\
\hline & 4 & & 0.710 & 34.4 & 40.9 & 95.0 & 11.1 & 77.8 \\
\hline
\end{tabular}

$A U C$ area under the curve value obtained by receiver-operating characteristic curve analysis, NA not analyzed due to PCR failure

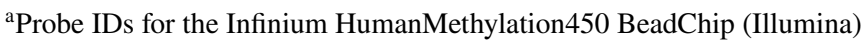

${ }^{\mathrm{b}}$ Infinium probe $\mathrm{CpG}$ sites and their neighboring $\mathrm{CpG}$ sites which were analyzed using pyrosequencing and numbered as shown in Table S2. Exact Infinium probe $\mathrm{CpG}$ sites are underlined

c"ER < non-ER", when the DNA methylation level of the sample was lower than the cut-off value, the sample was diagnosed as belonging to the ER group; "ER> non-ER", when the DNA methylation level of the sample was higher than the cut-off value, the sample was diagnosed as belonging to the ER group

${ }^{\mathrm{d}}$ Sensitivity is defined as the ratio of the number of tissue samples diagnosed as belonging to the ER group based on the criteria relative to the exact number of the patients belonging to the ER group

${ }^{\text {e}}$ Specificity is defined as the ratio of the number of tissue samples not diagnosed as belonging to the ER group using the criteria employed, relative to the exact number of patients belonging to the non-ER group

Table 3 The criteria combining the validated marker CpG sites, cg17206555 (position 1), cg02046247 (position 1), and cg19918599 (position 2 ), for prediction of early recurrence (ER) of pancreatic ductal adenocarcinoma

\begin{tabular}{|c|c|c|c|c|}
\hline \multirow{2}{*}{$\begin{array}{l}\text { Number of CpG sites satisfying the crite- } \\
\text { ria shown in Table } 2\end{array}$} & \multicolumn{2}{|l|}{ Initial cohort } & \multicolumn{2}{|l|}{ Validation cohort } \\
\hline & Sensitivity $^{\mathrm{a}}(\%)$ & Specificity $^{\mathrm{b}}(\%)$ & Sensitivity $^{\mathrm{a}}(\%)$ & Specificity $^{\mathrm{b}}(\%)$ \\
\hline One or more & 100 & 46.7 & 88.9 & 25.3 \\
\hline Two or three & 81.8 & 71.7 & 88.9 & 70.4 \\
\hline Three & 54.6 & 86.7 & 55.6 & 88.9 \\
\hline
\end{tabular}

${ }^{\text {a }}$ Sensitivity is defined as the ratio of the number of tissue samples diagnosed as belonging to the ER group based on the criteria relative to the exact number of the patients belonging to the ER group

${ }^{\mathrm{b}}$ Specificity is defined as the ratio of the number of tissue samples not diagnosed as belonging to the ER group using the criteria employed, relative to the exact number of patients belonging to the non-ER group 
revealed a significantly higher incidence of nuclear CDK14 immunoreactivity in PDACs in the ER group than that in the non-ER group (Figure S4). Even though sufficient sensitivity and specificity cannot be obtained solely by immunohistochemical examination, in a clinical setting, such examination using formalin-fixed paraffin-embedded tissue specimens of surgically resected material would be an auxiliary procedure for ER risk diagnosis based on quantification of DNA methylation.

CDK14 reportedly participates in the proliferation and invasion of many tumor cells, including those of breast cancer (Imawari et al. 2018), glioma (Fan et al. 2015), hepatocellular carcinoma (Tu et al. 2019), and ovarian cancer (Ou-Yang et al. 2017). In pancreatic cancers, $C D K 14$ has been revealed as a hub gene in the interaction network including the $\mathrm{Wnt} / \beta$-catenin pathway and phosphoinositide 3-kinase/Akt signaling pathway, and its increased expression is associated with poorer overall patient survival (Yuan et al. 2019). Although there are no published data to suggest that $C D K 14$ gene expression is regulated by DNA methylation, it is feasible that DNA hypomethylation might affect the risk of ER in PDAC through overexpression of $C D K 14$.

On the other hand, other marker CpG sites, position 1 of $\operatorname{cg} 02046247$ and position 2 of $\operatorname{cg} 19918599$, included in Table 3 are located outside $\mathrm{CpG}$ islands (open sea regions) in intergenic regions and would not participate in regulating the expression of specific genes. This is consistent with the results of our previous studies, indicating that even DNA methylation alterations at $\mathrm{CpG}$ sites not involved in the expression of functionally important genes would be potentially excellent surrogate markers for cancer diagnostics (Fujimoto et al. 2020; Nagashio et al. 2011). In general, most 5-methylcytosine residues seem to be controlled in a coordinated manner with their neighbors, rather than being independent, resulting in a domain wherein all $\mathrm{CpG}$ sites show largely similar methylation levels (Yokoyama et al. 2015). During carcinogenesis, the DNA methylation status of neighboring $\mathrm{CpG}$ sites included in the same domain is frequently altered en bloc (Johnstone et al. 2020). If such a domain includes important tumor-related genes whose function determines the ER of PDACs, it is feasible that the DNA methylation levels of neighboring $\mathrm{CpG}$ sites in the same domain could become surrogate markers for ER, even though they are not directly involved in regulation of specific genes.

In summary, we have identified potential biomarker $\mathrm{CpG}$ sites and established criteria that would be applicable to formalin-fixed paraffin-embedded tissue samples, and also to tumors both with and without an abundant cancer stroma consisting of infiltrating lymphocytes, fibroblasts, and other stromal cells, for prediction of ER of PDAC. Even though a large-scale prospective validation study is of course needed, such prognostication based on DNA methylation diagnostics may provide a breakthrough for personalized treatment of patients with aggressive PDAC. In addition, we have developed a system for quantification of DNA methylation involving high-performance liquid chromatography [HPLC], which is suitable for diagnosis of clinical samples containing various cell linages (Yotani et al. 2018). Using such appropriate diagnostic approaches, we expect that our markers would be applicable for prognostication using samples of pancreatic juice and blood including circulating tumors cells or cell-free DNA samples.

Supplementary Information The online version contains supplementary material available at https://doi.org/10.1007/s00432-021-03541-6.

Funding This study was supported by Funding for Research to Expedite Effective Drug Discovery by Government, Academia and Private Partnership (19ak0101043h0105) from the Japan Agency for Medical Research and Development (AMED), and also KAKENHI (16H02472 and 16K08720) from the Japan Society for the Promotion of Science.

Data availability The Infinium assay data have been deposited in the Gene Expression Omnibus (GEO) database (https://www.ncbi.nlm.nih. gov/geo/) (accession number: GSE155353).

\section{Compliance with ethical standards}

Conflict of interest The authors have no conflicts of interest to disclose.

Ethical approval This study was approved by the Ethics Committees of the National Cancer Center (ID 2004-033) and Keio University (ID 20040034, 20120280), in accordance with the ethical standards of the institutional research committees and with the Helsinki declaration.

Consent to participate Written informed consent was obtained from the patients to participate this research.

Consent for publication Written informed consent was obtained from the patients for publication.

Open Access This article is licensed under a Creative Commons Attribution 4.0 International License, which permits use, sharing, adaptation, distribution and reproduction in any medium or format, as long as you give appropriate credit to the original author(s) and the source, provide a link to the Creative Commons licence, and indicate if changes were made. The images or other third party material in this article are included in the article's Creative Commons licence, unless indicated otherwise in a credit line to the material. If material is not included in the article's Creative Commons licence and your intended use is not permitted by statutory regulation or exceeds the permitted use, you will need to obtain permission directly from the copyright holder. To view a copy of this licence, visit http://creativecommons.org/licenses/by/4.0/. 


\section{References}

Arai E, Ushijima S, Gotoh M et al (2009) Genome-wide DNA methylation profiles in liver tissue at the precancerous stage and in hepatocellular carcinoma. Int J Cancer 125:2854-2862

Arai E, Chiku S, Mori T et al (2012) Single-CpG-resolution methylome analysis identifies clinicopathologically aggressive $\mathrm{CpG}$ island methylator phenotype clear cell renal cell carcinomas. Carcinogenesis 33:1487-1493

Baylin SB, Jones PA (2016) Epigenetic determinants of cancer. Cold Spring Harb Perspect Biol 8:a019505

Bibikova M, Le J, Barnes B, Saedinia-Melnyk S et al (2009) Genomewide DNA methylation profiling using $\operatorname{Infinium(R)}$ assay. Epigenomics 1:177-200

Brierley JD, Gospodarowicz MK, Wittekind C (2017) Stomach. TNM Classification of Malignant Tumours, 8th edn. Wiley-Blackwell Press, Hoboken, pp 93-95

Conroy T, Hammel P, Hebbar M et al (2018) FOLFIRINOX or gemcitabine as adjuvant therapy for pancreatic cancer. New Eng J Med 379:2395-2406

Egawa S, Toma H, Ohigashi H et al (2012) Japan pancreatic cancer registry; 30th year anniversary: Japan Pancreas Society. Pancreas 41:985-992

Fan S, Zhao C, Zhang L et al (2015) Knockdown of PFTK1 inhibits the migration of glioma cells. J Mol Neurosci 57:257-264

Fujimoto M, Arai E, Tsumura K et al (2020) Establishment of diagnostic criteria for upper urinary tract urothelial carcinoma based on genome-wide DNA methylation analysis. Epigenetics 4:1-13

Gotoh M, Arai E, Wakai-Ushijima S et al. (2011) Diagnosis and prognostication of ductal adenocarcinomas of the pancreas based on genome-wide DNA methylation profiling by bacterial artificial chromosome array-based methylated $\mathrm{CpG}$ island amplification. J Biomed Biotechnol 2011;2011:780836. https://doi. org/10.1155/2011/780836

Groot VP, Rezaee N, Wu W et al (2018) Patterns, timing, and predictors of recurrence following pancreatectomy for pancreatic ductal adenocarcinoma. Ann Surg 267:936-945

Groot VP, Gemenetzis G, Blair AB et al (2019) Defining and predicting early recurrence in 957 patients with resected pancreatic ductal adenocarcinoma. Ann Surg 269:1154-1162

Hassan MM, Bondy ML, Wolff RA et al (2007) Risk factors for pancreatic cancer: case-control study. Am J Gastroenterol 102:2696-2707

Hruban RH, Maitra A, Kern SE et al (1997) Precursors to pancreatic cancer. Gastroenterol Clin North Am 36:831-849

Hruban RH, Boffetta P, Hiraoka N et al (2019) Tumours of the pancreas. WHO Classification of Tumours of Digestive System Tumours, 5th edn. IARC Press, Lyon, pp 279-337

Imawari Y, Mimoto R, Hirooka S et al (2018) Downregulation of dualspecificity tyrosine-regulated kinase 2 promotes tumor cell proliferation and invasion by enhancing cyclin-dependent kinase 14 expression in breast cancer. Cancer Sci 109:363-372

Johnstone SE, Reyes A, Qi Y et al (2020) Large-scale topological changes restrain malignant progression in colorectal cancer. Cell 182:1474-1489

Jones PA, Issa JP, Baylin S (2016) Targeting the cancer epigenome for therapy. Nat Rev Genet 17:630-641

Kanai Y, Nishihara H, Miyagi Y et al (2018) The Japanese Society of Pathology Guidelines on the handling of pathological tissue samples for genomic research: standard operating procedures based on empirical analyses. Pathol Int 68:63-90

Kim J, Bretz CL, Lee S (2015) Epigenetic instability of imprinted genes in human cancers. Nucleic Acids Res 43:10689-10699
Kurahara H, Maemura K, Mataki Y et al (2018) A therapeutic strategy for resectable pancreatic cancer based on risk factors of early recurrence. Pancreas 47:753-758

Kuramoto J, Arai E, Tian Y (2017) Genome-wide DNA methylation analysis during non-alcoholic steatohepatitis-related multistage hepatocarcinogenesis: comparison with hepatitis virus-related carcinogenesis. Carcinogenesis 38:261-270

Liu Y, Gu Y, Su M et al (2019) An analysis about heterogeneity among cancers based on the DNA methylation patterns. BMC Cancer 19:1259

Makabe T, Arai E, Hirano T et al (2019) Genome-wide DNA methylation profile of early-onset endometrial cancer: its correlation with genetic aberrations and comparison with late-onset endometrial cancer. Carcinogenesis 40:611-623

Matsumoto I, Murakami Y, Shinzeki M et al (2015) Proposed preoperative risk factors for early recurrence in patients with resectable pancreatic ductal adenocarcinoma after surgical resection: a multicenter retrospective study. Pancreatology 15:674-680

Mishra SR, Mahapatra KK, Behera BR et al. (2020) Inflammasomes in cancer: Effect of epigenetic and autophagic modulations. Semin Cancer Biol 9:S1044-579X(20)30205-4.

Motoi F, Satoi S, Honda G et al (2019) A single-arm, phase II trial of neoadjuvant gemcitabine and $\mathrm{S} 1$ in patients with resectable and borderline resectable pancreatic adenocarcinoma: PREP-01 study. J Gastroenterol 54:194-203

Murphy JE, Wo JY, Ryan DP et al (2018) Total neoadjuvant therapy with FOLFIRINOX followed by individualized chemoradiotherapy for borderline resectable pancreatic adenocarcinoma: a phase 2 clinical trial. JAMA Oncol 4:963-969

Nagashio R, Arai E, Ojima H et al (2011) Carcinogenetic risk estimation based on quantification of DNA methylation levels in liver tissue at the precancerous stage. Int J Cancer 129:1170-1179

Neoptolemos JP, Palmer DH, Ghaneh P et al (2017) Comparison of adjuvant gemcitabine and capecitabine with gemcitabine monotherapy in patients with resected pancreatic cancer (ESPAC-4): a multicentre, open-label, randomised, phase 3 trial. Lancet 389:1011-1024

Nishio K, Kimura K, Amano R et al (2017) Preoperative predictors for early recurrence of resectable pancreatic cancer. World $\mathrm{J}$ Surg Oncol 15:16

Nones K, Waddell N, Song S et al (2014) Genome-wide DNA methylation patterns in pancreatic ductal adenocarcinoma reveal epigenetic deregulation of SLIT-ROBO, ITGA2 and MET signaling. Int J Cancer 135:1110-1118

Ohara K, Arai E, Takahashi Y et al (2017) Genes involved in development and differentiation are commonly methylated in cancers derived from multiple organs: a single-institutional methylome analysis using 1007 tissue specimens. Carcinogenesis $38: 241-251$

Ou-Yang J, Huang LH, Sun XX (2017) Cyclin-dependent kinase 14 promotes cell proliferation, migration and invasion in ovarian cancer by inhibiting Wnt signaling pathway. Gynecol Obstet Invest 82:230-239

Peng DF, Kanai Y, Sawada M et al (2005) Increased DNA methyltransferase 1 (DNMT1) protein expression in precancerous conditions and ductal carcinomas of the pancreas. Cancer Sci 96:403-408

Peng DF, Kanai Y, Sawada M et al (2006) DNA methylation of multiple tumor-related genes in association with overexpression of DNA methyltransferase 1 (DNMT1) during multistage carcinogenesis of the pancreas. Carcinogenesis 27:1160-1168

Sato T, Arai E, Kohno T et al (2014) Epigenetic clustering of lung adenocarcinomas based on DNA methylation profiles in adjacent lung tissue: Its correlation with smoking history and chronic obstructive pulmonary disease. Int J Cancer 135:319-334

Siegel RL, Miller KD, Jemal A (2016) Cancer statistics, 2016. CA Cancer J Clin 66:7-30 
Sinn M, Bahra M, Liersch T et al (2017) CONKO-005: Adjuvant chemotherapy with gemcitabine plus erlotinib versus gemcitabine alone in patients after $\mathrm{R} 0$ resection of pancreatic cancer: a multicenter randomized phase III trial. J Clin Oncol 35:3330-3337

Sugiura T, Uesaka K, Kanemoto H et al (2012) Serum CA19-9 is a significant predictor among preoperative parameters for early recurrence after resection of pancreatic adenocarcinoma. J Gastrointest Surg 16:977-985

Tsumura K, Arai E, Tian Y et al (2019) Establishment of permutation for cancer risk estimation in the urothelium based on genomewide DNA methylation analysis. Carcinogenesis 40:1308-1319

Tu J, Zhao Z, Xu M et al (2019) LINC00707 contributes to hepatocellular carcinoma progression via sponging miR-206 to increase CDK14. J Cell Physiol 234:10615-10624

Uesaka K, Boku N, Fukutomi A et al (2016) Adjuvant chemotherapy of S-1 versus gemcitabine for resected pancreatic cancer: a phase 3, open-label, randomised, non-inferiority trial (JASPAC 01). Lancet 388:248-257

Wen X, Jeong S, Kim Y et al (2017) Improved results of LINE-1 methylation analysis in formalin-fixed, paraffin- embedded tissues with the application of a heating step during the DNA extraction process. Clin Epigenetics 9:1

Yamanoi K, Arai E, Tian Y et al (2015) Epigenetic clustering of gastric carcinomas based on DNA methylation profiles at the precancerous stage: its correlation with tumor aggressiveness and patient outcome. Carcinogenesis 36:509-520

Yokoyama T, Miura F, Araki H et al (2015) Changepoint detection in base-resolution methylome data reveals a robust signature of methylated domain landscape. BMC Genomics 16:594

Yotani T, Yamada Y, Arai E et al (2018) Novel method for DNA methylation analysis using high-performance liquid chromatography and its clinical application. Cancer Sci 109:1690-1700

Yuan YH, Zhou J, Zhang Y et al (2019) Identification of key genes and pathways downstream of the $\beta$-catenin-TCF7L1 complex in pancreatic cancer cells using bioinformatics analysis. Oncol Lett 18:1117-1132

Zhang J, Shi K, Huang W et al (2019) The DNA methylation profile of non-coding RNAs improves prognosis prediction for pancreatic adenocarcinoma. Cancer Cell Int 19:107

Publisher's Note Springer Nature remains neutral with regard to jurisdictional claims in published maps and institutional affiliations. 